\title{
Updates on Urgency of Treating Stroke
}

\author{
Adel Ekladious*1,2 \\ ${ }^{1}$ Bega -Southeast Regional Hospital, 4 Virginia Drive, Bega NSW 2550, Western Australia, Australia \\ ${ }^{2}$ Associate Professor, Faculty of Health and Medical sciences, University of Western Australia, Western Australia, Australia
}

*Corresponding author: Adel Ekladious, Associate Professor, Faculty of Health and Medical sciences, University of Western

Australia, 35 Stirling Hwy, Crawley, Western Australia, Australia, Email: ekladiou@hotmail.com

\section{ARTICLE INFO}

Received: 幽 August 12, 2021

Published: 蔧 August 19, 2021

\section{ABSTRACT}

Citation: Adel Ekladious. Updates on Urgency of Treating Stroke. Biomed J Sci \& Tech Res 38(2)-2021. BJSTR. MS.ID.006126.

\section{Introduction}

It is a proof of concept that time is muscle in treating myocardial infarction and even if for any reason you missed the boat in timely management of heart attack, we postulate that probably you might have a second chance which is heart transplant if patient meets the requirement, and this is not an excuse for not treating the heart attack very urgently [1]. The situation in stroke is more serious and more urgent [2] as you need to exclude stroke mimics in a very short time to make a therapeutic decision, in a large vessel stroke [middle cerebral artery stroke]. There is a loss of 2 million nerve cells per each minute delay [3] which is correlated with loss of 1.8 days of healthy life [3], After saucerful treatment of heart attack you can still run a marathon in contrast of stroke that you might need to lose few brain cells to be in a vegetative state. Most of strokes are ischemic, around $80 \%$, less than $15 \%$ are hemorrhagic, and less than $2 \%$ are venous stroke (cortical or cerebral venous stroke) [4]. Treatment of each type of stroke is quite different, and all types of strokes is emergency and needs to confirm the diagnosis.

\section{Mini Review}

In this review, i am going to discuss the updates of investigation and specific physical signs and symptoms which help to reach a correct diagnosis in a very short time, and iniate urgent treatment. Firstly, diagnosis of stroke and ruling out stroke mimics is of para-amount importance, Seizure, manganous Aurea, cardiogenic and metabolic syncope, encephalitis, demyelination, infective endocarditis and embolic stroke, metabolic encephalopathy, need to be ruled out some tips to exclude mimics. a) Seizure, loss of consciousness, patient can't remember what happened, post ictal state, Shaking, bite on the lateral side of the tongue, if patient has history of seizure, usually most seizure is stereotyped, patient can have Todd's paralysis which usually resolve in few hours [5].

b) Migranous Aurea, history of migraine, fortification spectra, Aurea could be in language and pronunciation, sensory, motor, usually it is slowly progressing, each one modality happened in one time then followed by another modality, patient could have nausea [6], patient with basilar migraine might have ptosis or Horner syndrome [7].

c) Syncope, quite sudden, patient lose his consciousness, medical history usually includes cardiac problems, familial history of cardiac syncope or sudden death (familial cardiomyopathy, Q-T syndrome).

d) Loss of consciousness usually last for seconds, if it is more than few minutes, other diagnosis should be considered [8], most of these patients have car accidents during syncope.

e) Transient Global Amnesia, it is sudden, temporary episode of memory loss, recall of recent events vanishes, sometimes patient can't remember anything about the event and keeps asking same questions [8].

f) Encephalitis, usually patient will be confused and have diseases or sepsis underlying problems like chronic liver disease, respiratory failure, brain infection. 
g) Demyelination, crescendo is slowly, commonly affecting females, usually resolve in few days, could be manifested as a flare of multiple sclerosis or could be precipitated by infection, if associated with infection, clinician should treat the infection and not to give methyl prednisolone [9].

Sometimes confirming the diagnosis of stroke will be difficult, here we are presenting other tools which help the diagnosis of stroke and the pathology of stroke to help starting an early treatment.

1. The definition of TIA as clinical symptoms which last less than 24 hours is outdated [10] because MRI can confirm a diagnosis of stroke even if the clinical symptoms last less than 60 minutes [11].

2. Restriction diffusion in DWI can diagnose stroke even after one minute of having the symptoms and cytotoxic oedema can be confirmed by ADC [12].

3. Biomarkers in Ischemic Stroke, S100B and specific enolase are proteins measured from serum and correlate very well with the volume of stroke 24-27 hours [13]. As we treat ischemic stroke with Alteplase [single chain recombinant tissue plasminogen activator] in therapeutic window which is four and half hours, CT perfusion and CTA are a standard of care in Australian hospitals which usually show the volume of infarct tissue (core) and the volume of hypo perfused region that can be salvageable with reperfusion(penumbra), even the severely hypo perfused region with good collateral below the occluded vessels, still have a high chance of reperfusion [13]. More than third of stroke are excluded from lysis because of the unknown time of symptoms. It became a best practice in Australia to consider the mismatch between DWI-Flair Mismatch.

Is a surrogate marker of the time of onset of stroke [14] specially in wake-up stroke?. Endovascular therapy is a standard of care for proximal large vessel occlusion, therapeutic window is up to 12 hours [15]. Venous stroke is an emergency, it affects mostly females, usually presented with seizure, decreased level of consciousness due diffuse encephalopathy, signs of increased intracranial pressure, headache, cranial nerve palsy, It is underdiagnosed because of the rarity of the stroke.

\section{Predisposing Factors}

Female gender, contraceptive pills, dehydration, inherited thrombophilia, myeloproliferative diseases, Rare diseases such as paroxysmal nocturnal hemoglobinuria. Helpful serum markers are positive D dimer, Uncontracted CT shows dense clot sign in cerebral vein or sinus, CT venogram is diagnostic. Due to increased intracranial tension, CT might show cerebral oedema, mass effect and intracranial hemorrhage which can progress to subarachnoid hemorrhage and massive hemorrhagic infarction [16]. Treatment is mainly anticoagulation, patients who do not respond to anticoagulation, treatment should be escalated to endovascular thrombolysis [17]. Hemorrhagic stroke is a life-threatening emergency, usually patient manifest with decline in consciousness and very mild neurological loss, seizure and signs of intracranial hypertension. Mortality is high in the first 90 days. Types of hemorrhagic stroke either lobar hemorrhage due to amyloid angiopathy, hypertensive bleed in Basel ganglia, hypothalamus and brain stem, subarachnoid hemorrhage due to ruptured Aneurysm, anticoagulation, AVM misinformation, cocaine abuse CTA is diagnostic as it shows Spot sign which is marked attenuation due to active bleed [18]. There is no definite cure of hemorrhagic stroke, patient should be managed in ICU to observe and treat seizures, intracranial hypertension, systolic hypertension with aim under 140. Treating fever, sepsis, hyperglycemia, endovascular treatment for ruptured aneurism, Reversing anticoagulation, prophylaxis for thrombosis with pneumatic compression in the first few days followed by low molecular weight heparin, craniectomy for evacuation of Hematoma, external ventricular drain for intraventricular hemorrhage causing hydrocephalus [19].

\section{Conflict of Interest}

There is no conflict of interest.

\section{References}

1. Zimmer RJ, Lee MS (2010) Transplant coronary heart disease. JACC cardiovasc Interv pp. 367-377.

2. Peter M Fernandes, William N Whiteley. Simon R Hart, Rustam Al-Shahi Salmon (2013) Stroke: mimics and Chameleons. Pract Neurol 13(1): 2128.

3. Atte Meretoja, Mahsa Keshtkaran, Jeffrey L Saver, Turgut Tatlisumak, Mark W Parsons, et al. (2014) Stroke thrombolysis: save a minute. save a day. Stroke 45: 1053-1058.

4. Saposnik G, Barinagarre F, Brownmenteria RD, Bushnell CD, Cucchiara B, et al. (2011) American heart Association Stroke council on epidemiology and prevention. Diagnosis and management of cerebral venous thrombosis: a statement for healthcare professionals from American heart association. Stroke 42: 1158-1192.

5. Doudoux H, Fournier M, Vercueil L (2020) Postictal syndrome:the forgotton Continent, an overview of the clinical, biochemical and imaging features, Rev Neurol( Paris)176(1-2): 62-74.

6. PB Croft, KWG Heathfield, M Swash (1973) Differential Diagnosis of Transient Amnesia.Br Med J 4: 593.

7. Kanieck RG (2009) Basilar - type Migraine. Curr pain Headache Rep 13(3): 217-220.

8. Miller JW, Petersen RC, Metter EJ, Millikan CH, Yanagihara T (1987) transient Global Amnesia: Clinical Characteristics and prognosis. Neurology 37: 733-737.

9. W I McDonald, A Compston, G Edan, D Goodkin, H P Hartung, et al. (2001) Recommended diagnostic Criteria for multiple Scelrosis: Guidelines from The International Panel on the diagnosis of Multiple Sclerosis. Ann Neurol pp.121-127.

10. Gennai S, Giordano-Orsini G,lefour S, Cuisenier P (2018) ( Transient Ischemic Attack: limits. and Challenges of Early Management), Presse Med47(11-12 Pt 1): 934-937. 
11. Cereda CW, Olivot JM (2018) Emergency Department (ED) triage for transient ischemic attack (TIA). Curr Atherosclerosis Rep 20(11): 56.

12. Schellinger PD (2005) The evolving role of advanced MR imaging as a Management tool for adult ischemic stroke: Awestern -European prespective. Neuroimaging Clin N Am 15: 245-258.

13. L Persson, H G Hårdemark, J Gustafsson, G Rundström, I Mendel-Hartvig et al. (1987) S100 Protein and neuron specific enolase in cerebrospinal fluid and serum: markers of Cell damage in human central nervous system. Stroke 18(5): 911-918.

14. Beauchamp NJ, Barker PB, Wang PY, Van Zijl PC (1999) Imaging of acute cerebral. ischemia. Radiology 212: 307-324.

15. Amrou Sarraj, Navdeep Sangha, Muhammad Shazam Hussain, Dolora Wisco, Nirav Vora, etal. (2016) Endovascular Therapy for Acute Ischemic Stroke with Occlusion of the Middle Cerebral Artery M2 Segment.JAMA Neurol 73(11): 1291-1296.

ISSN: 2574-1241

DOI: 10.26717/BJSTR.2021.38.006126

Adel Ekladious. Biomed J Sci \& Tech Res

(c) (P) This work is licensed under Creative

Submission Link: https://biomedres.us/submit-manuscript.php
16. Zheng H, Chen C, Zhang J, Hu Z (2016) Mechanism and Therapy of Brain Edema after Intracerebral Hemorrhage. Cerbrovas Dis 42: 155-169.

17. K Einhaupl, J Stam, MG Bousser, SF de Bruijn, JM Ferro, et al. (2010) EENS guidelines on the treatment of cerebral venous and sinus thrombosis in adult patients. EUR J Neurol 10(2010): 1299-1235.

18. Andrew M Demchuk, Dar Dowlatshahi, David Rodriguez-Luna, Carlos A Molina, Yolanda Silva Blas, et al. (2012) Prediction of hematoma growth and outcome in patients with intracerebral Hemorrhage using the CT Angiography spot sign (PREDICT): A Prospective Observational study. The Lancet Neurology 11 (4): 307-314.

19. Guillaume Saliou, Gaelle Paradot, Catherine Gondry, Roger Bouzerar, Pierre Lehmann, et al. (2021) A phase - contrast mri Study of acute and chronic hydrodynamic alterations after hydrocephalus induced by subarachnoid hemorrhage. Journal of Neuroimaging 22(4): 343-350.

$\begin{array}{ll}\text { BIOMEDICAL } & \text { Assets of Publishing with us } \\ \text { RESEARCHES } & \text { - Global archiving of articles } \\ \text { - Immediate, unrestricted online access }\end{array}$

